\title{
An Innovative Method for Robots Modeling and Simulation
}

\author{
Laura Celentano \\ Dipartimento di Informatica e Sistemistica \\ Università degli Studi di Napoli Federico II \\ Napoli, \\ Italy
}

\section{Introduction}

The robot dynamics modeling and simulation problem has been studied for the last three decades intensively.

In particular, the forward dynamics problem of a robot is a very relevant issue, which there is still to say about in terms of efficient computation algorithms, that can be also simple to understand, to develop and to implement, above all for practical robots, robots with many links and/or with flexible links (Featherstone, 1987), (Featherstone \& Orin, 2000), (Sciavicco \& Siciliano, 2000), (Khalil \& Dombre, 2002).

Indeed, in these cases the methods based on the classical Lagrange formulation give rise to an analytical model with numerous terms that may be difficult to use. The methods based on the Newton-Euler formulation are not very easy to apply and do not provide easily manageable analytical formulae, even if they are efficient from a computational point of view (Featherstone, 1987).

An important contribution to solve the previous problems is given (Celentano, 2006), (Celentano \& Iervolino, 2006), (Celentano \& Iervolino, 2007) by a new, simple and efficient methodology of analysis, valid for all of robots, that makes use of a mathematical model containing a lower number of algebraic terms and that allows computing, with a prescribed maximum error, the gradient of the kinetic energy starting from the numerical knowledge of the only inertia matrix rather than using, as usually found in the literature, complex analytical calculations of the closed-form expression of this matrix.

@ This result is very strong because it allows solving the forward dynamics problem of a robot in a simple and efficient manner, by analytically or numerically computing the inertia matrix and the potential energy gradient only.

Moreover, this method allows students, researchers and professionals, with no particular knowledge of mechanics, to easily model planar and spatial robots with practical links.

From this methodology follows also a simple and efficient algorithm for modeling flexible robots dividing the links into rigid sublinks interconnected by equivalent elastic joints and approximating and/or neglecting some terms related to the deformation variables.

In this chapter some of the main results stated in (Celentano, 2006), (Celentano \& Iervolino, 2006), (Celentano \& Iervolino, 2007) are reported.

Source: New Approaches in Automation and Robotics, Book edited by: Harald Aschemann, ISBN 978-3-902613-26-4, pp. 392, May 2008, I-Tech Education and Publishing, Vienna, Austria 
In details, in Section II the new integration scheme for robots modeling, based on the knowledge of the inertia matrix and of the potential energy only, is reported (Celentano \& Iervolino, 2006).

In Section III, for planar robots with revolute joints, theorems can be introduced and demonstrated to provide a sufficiently simple and efficient method of expressing both the inertia matrix and the gradient of the kinetic energy in a closed and elegant analytical form. Moreover, the efficiency of the proposed method is compared to the efficiency of the Articulated-Body method, considered one of the most efficient Newtonian methods in the literature (Celentano \& Iervolino, 2006).

In Section IV, for spatial robots with generic shape links and connected, for the sake of brevity, with spherical joints, several theorems are formulated and demonstrated in a simple manner and some algorithms that allow efficiently computing, analytically the inertia matrix, analytically or numerically the gradient of the kinetic and of the gravitational energy are provided. Furthermore, also in this case a comparison of the proposed method in terms of efficiency with the Articulated-Body one is reported (Celentano \& Iervolino, 2007). In Section V some elements of flexible robots modeling, that allow obtaining, quite simply, accurate and efficient, from a computational point of view, finite-dimensional models, are provided. Moreover, a significant example of implementation of the proposed results is presented (Celentano, 2007).

Finally, in Section VI some conclusions and future developments are reported.

\section{A new formulation of the Euler-Lagrange equation}

In the following a new formulation of the dynamic model of a robot mechanism in a more efficient form (for its analytical and/or numerical study) is presented.

It is well known that the usual form of the Euler-Lagrange equation for a generic robot with $n$ degrees of freedom, which takes on the form (De Wit et al., 1997)

$$
\frac{d}{d t} \frac{\partial T}{\partial \dot{q}}-\frac{\partial T}{\partial q}=r+\eta+u_{a}+u
$$

where: $q$ is the vector of the Lagrangian coordinates; $T$ is the kinetic energy, given by $T=\frac{1}{2} \dot{q}^{T} B(q) \dot{q}$, being $B$ the robot inertia matrix; $\gamma$ is the vector of the gravity forces, $\eta$ is the vector of the elasticity forces and $u_{a}$ is the non-conservative generalised forces (e.g. damping torques), which are usually function of $q$ and $\dot{q}$ only, and $u$ is the vector of the actuation generalised forces, is typically rewritten as

$$
B(q) \ddot{q}+C(q, \dot{q}) \dot{q}=\gamma+\eta+u_{a}+u,
$$

in which an expression of matrix $C$ is the following:

$$
C(q, \dot{q})=\sum_{i=1}^{n} \frac{\partial B}{\partial q_{i}} \dot{q}_{i}-\frac{1}{2}\left[\begin{array}{c}
\dot{q}^{T} \frac{\partial B}{\partial q_{1}} \\
\vdots \\
\dot{q}^{T} \frac{\partial B}{\partial q_{n}}
\end{array}\right] .
$$


By setting $B=\left\{b_{i j}\right\}, C=\left\{c_{i j}\right\}$, it is also

$$
c_{i j}=\sum_{k=1}^{n}\left(\frac{\partial b_{i j}}{\partial q_{k}}-\frac{1}{2} \frac{\partial b_{i k}}{\partial q_{i}}\right) \dot{q}_{k} .
$$

Alternatively, an equivalent matrix $C_{c}=\left\{c_{c i j}\right\}$, i.e. such that $C_{c} \dot{q}=C \dot{q}$, that makes uses of the Christoffell's symbols (Sciavicco \& Siciliano, 2000), is given by

$$
c_{c i j}=\sum_{k=1}^{n} \frac{1}{2}\left(\frac{\partial b_{i j}}{\partial q_{k}}+\frac{\partial b_{i k}}{\partial q_{j}}-\frac{\partial b_{j k}}{\partial q_{i}}\right) \dot{q}_{k} .
$$

There are various methods to calculate both $B(q)$ and $C(q, \dot{q})$. The simplest one, from a conceptual point of view, based on analytical expressions, is extremely complex and onerous, even if symbolic manipulation languages are employed. Other methods do require a more in-depth knowledge of mechanics (De Wit et al., 1997).

The next theorem provides an innovative, efficient and simple method for modeling and simulating a robot.

Theorem 1. If the Euler-Lagrange equation is rearranged as follows:

$$
\frac{d}{d t}(B \dot{q})=c+\gamma+\eta+u_{a}+u
$$

where $c$ is the gradient of the kinetic energy with respect to $q$, namely

$$
c=\frac{\partial T}{\partial q}
$$

then it can be efficiently integrated according to the innovative integration scheme of Fig. 1, where $\dot{q}_{g}=B \dot{q}$ and $F$ is a suitable function that allows computing $\dot{q}$ and $\ddot{q}_{g}$ from $u, q$ and $\dot{q}_{g}$.

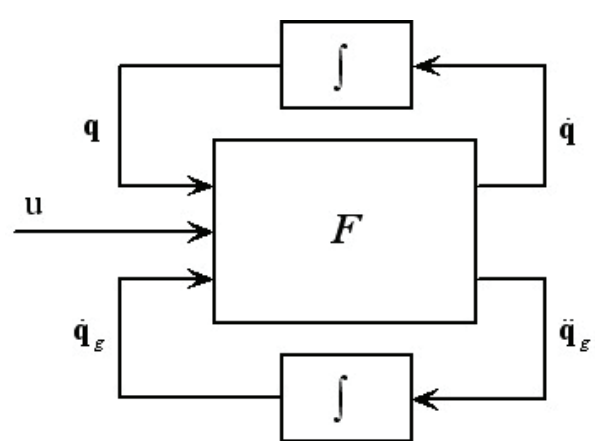

Fig. 1. Block diagram for the robot forward dynamics integration.

Proof. It is easy to verify that the algorithm that describe the function $F$ of the block scheme of Fig. 1 is: 
Step 1. Compute $B(q)$ by applying one of the classic methods proposed in the literature (see also Theorems 2 and 3 and (30) in Section 3).

Step 2. Compute $\dot{q}$ through the relationship $\dot{q}=B^{-1} \dot{q}_{g}$.

Step 3. Compute $c(q, \dot{q})$ and $u_{a}$.

Step 4. Compute $\frac{d}{d t}(B \dot{q})=\ddot{q}_{g}=c+\gamma+\eta+u_{a}+u$.

Remark 1. The proposed method allows avoiding the computation of the first term on the right hand-side of (4) or of the first two terms on the right-hand side of (5). Such saving is significant because the computational burden relative to this term is not negligible with respect to the one required by the calculation of $B^{-1} \dot{q}_{g}$.

This clearly emerges from Fig. 2, where the percentages of saved flops vs. the number of flops required to compute $B^{-1} \dot{q}_{g}$ are evaluated (in MATLAB ${ }^{\mathrm{TM}}$ environment) with reference to the coefficients in (4), for a considerable number of cases of robots with random and anyhow realistic links parameters.

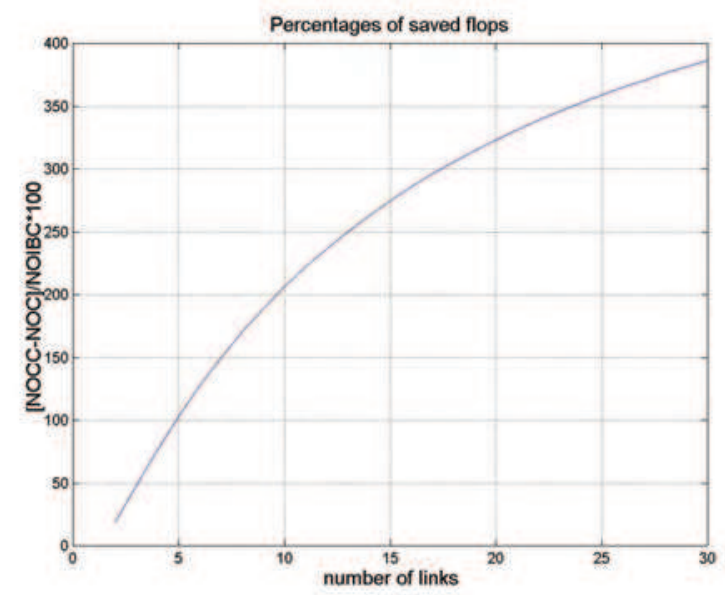

Fig. 2. Percentages of saved flops evaluated for robot models with random links parameters.

Remark 2. Since many efficient algorithms for the numerical computation of the matrix $B$ are available in the literature, the gradient of the kinetic energy $c$ can be computed in a very simple and accurate way numerically, instead of using a symbolic expression.

To this end, since the kinetic energy for the majority of the robots is a quadratic function respect to $\dot{q}$, whose coefficients are constant with respect to $q$ or depend on $q_{i}$ according to terms of the type $\sin \left(a q_{i}+\varphi\right)$, the following lemma is useful.

Lemma 1. The derivative of $\sin \left(a q_{i}\right)$ can be numerically computed by a two [three] points formula:

$$
\frac{d}{d q_{i}} \sin \left(a q_{i}\right)=\frac{\sin \left[a\left(q_{i}+\Delta\right)\right]-\sin \left(a q_{i}\right)}{\Delta}\left[\frac{d}{d q_{i}} \sin \left(a q_{i}\right)=\frac{\sin \left[a\left(q_{i}+\Delta\right)\right]-\sin \left[a\left(q_{i}-\Delta\right)\right]}{2 \Delta}\right]
$$


with error:

$$
\begin{gathered}
e_{2}=-\frac{1}{2} a^{2} \sin (a \chi) \Delta,\left|e_{2}\right| \leq a^{2} \frac{\Delta}{2}, \quad \chi \in\left(q_{i}, q_{i}+\Delta\right) \\
{\left[e_{3}=-\frac{1}{6} \Delta^{2} a^{3} \cos (a \chi),\left|e_{3}\right| \leq a^{3} \frac{\Delta^{2}}{6}, \chi \in\left(q_{i}-\Delta, q_{i}+\Delta\right)\right] .}
\end{gathered}
$$

Proof. See any numerical computation book.

In view of Lemma 1 it is possible to compute, with a prescribed maximum error, the gradient of the kinetic energy starting from the numerical knowledge of the inertia matrix $B$ rather than using, as usually found in literature, complex analytical calculations of the analytical expression of $B$. In details, for practical precision purposes, a good value of $\partial T / \partial q$ can be obtained using a two [three] points formula with $\Delta=10^{-3} \div 10^{-4}$ [with $\left.\Delta=10^{-2} \div 10^{-3}\right]$ and evaluating $n-1[2(n-1)]$ times the inertia matrix $B$ numerically.

\section{Planar robot modeling}

In the case of planar robots with revolute joints, theorems can be introduced and demonstrated to provide a sufficiently simple and efficient method of expressing both the inertia matrix and the gradient of the kinetic energy in a closed and elegant analytical form.

\subsection{Hypotheses and notations}

In the following it is considered the case of a generalised planar robot constituted by $n$ links, each of them with parallel rotation axes $C^{-}, C^{+}$and center of mass $G$ belonging to the plane containing the two relative rotation axes (see Fig. 3). In Fig. 4 a planar robot with three links and a horizontal work plane is shown.

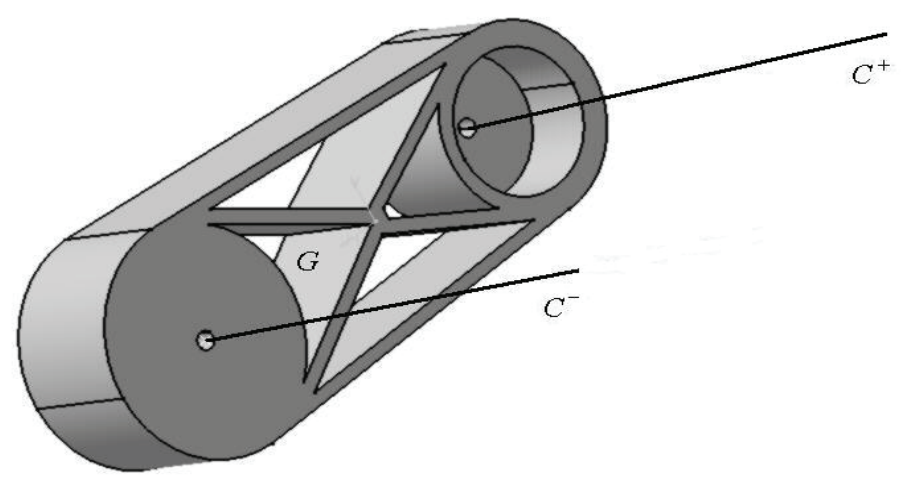

Fig. 3. The generic link of the considered generalised planar robot. 


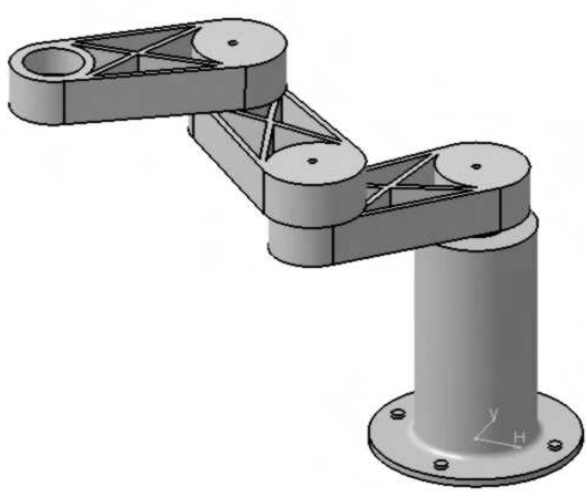

Fig. 4. The considered generalised planar robot with three links and horizontal work plane. Referring to Figs. 5 and 6 the next notations are also employed:

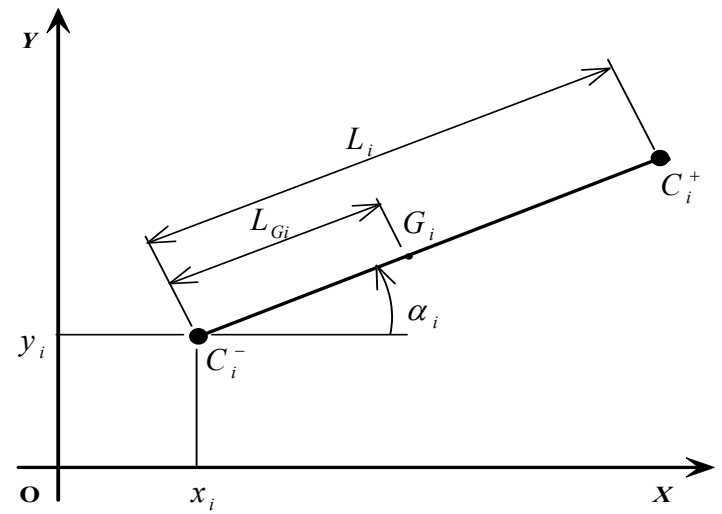

Fig. 5. Geometric characteristics of the $i$-th link.

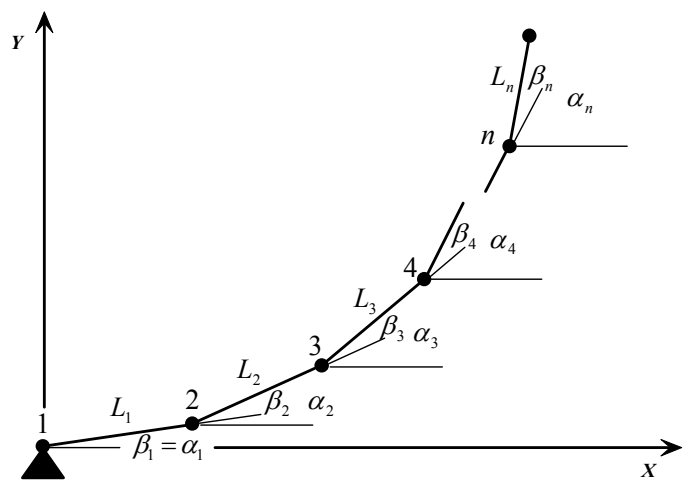

Fig. 6. Schematic representation of a planar robot with $n$ links. 
$q_{i}=\left[\begin{array}{lll}x_{i} & y_{i} & \alpha_{i}\end{array}\right]^{T}=$ absolute coordinates of the $i$-th link,

$I_{i} \quad=$ inertia matrix of the $i$-th link in terms of the coordinates $q_{i}$,

$M_{i} \quad=$ total mass of the $i$-th link,

$J_{i}, \rho_{i} \quad=$ inertia moment and radius of the $i$-th link with respect to the rotation axis $C_{i}^{-}$,

$N_{i} \quad=$ static moment of the $i$-th link with respect to the plane containing $C_{i}^{-}$and orthogonal to the plane passing through $C_{i}^{-}$and $C_{i}^{+}$,

$L_{G i} \quad=$ distance of the center of mass $G_{i}$ of the $i$-th link from the axis $C_{i}^{-}$,

$\alpha_{1 \ldots i}=\left[\begin{array}{llll}\alpha_{1} & \alpha_{2} & \ldots & \alpha_{i}\end{array}\right]^{T}=$ vector of the absolute angular coordinates of the chain constituted by the first $i$ links,

$\beta_{1 \ldots i}=\left[\beta_{1} \beta_{2} \ldots \beta_{i}\right]^{T}=$ vector of the relative angular coordinates of the chain constituted by the first $i$ links,

$A_{i} \quad=$ inertia matrix of the $i$-th link in terms of the coordinates $\alpha_{1 \ldots i}$,

$B_{i} \quad=$ inertia matrix of the $i$-th link in terms of the coordinates $\beta_{1 \ldots i}$,

$\alpha=\left[\begin{array}{llll}\alpha_{1} & \alpha_{2} & \ldots & \alpha_{n}\end{array}\right]^{T}=$ vector of the absolute angular coordinates of the robot,

$\beta=\left[\beta_{1} \beta_{2} \ldots \beta_{n}\right]^{T}=$ vector of the relative angular coordinates of the robot,

A $\quad=$ inertia matrix of the robot in terms of the coordinates $\alpha$,

$B=$ inertia matrix of the robot in terms of the coordinates $\beta$.

\subsection{Main results}

Consider a generic link of a planar robot, with revolute joints. The following theorem can be stated.

Theorem 2. The kinetic energy $T_{i}$ of the link of a planar robot, whose rotation axes are parallel and center of mass belongs to the plane that contains the respective rotation axes (see Figs. 3 and 5) can be calculated by the relationship

$$
T_{i}=\frac{1}{2} \dot{q}_{i}^{T} I_{i} \dot{q}_{i}
$$

where

$$
I_{i}=\left[\begin{array}{ccc}
M_{i} & 0 & -N_{i} \sin \alpha_{i} \\
0 & M_{i} & N_{i} \cos \alpha \\
-N_{i} \sin \alpha_{i} & N_{i} \cos \alpha_{i} & J_{i}
\end{array}\right]=M_{i}\left[\begin{array}{ccc}
1 & 0 & -L_{G i} \sin \alpha_{i} \\
0 & 1 & L_{G i} \cos \alpha_{i} \\
-L_{G i} \sin \alpha_{i} & L_{G i} \cos \alpha_{i} & \rho_{i}^{2}
\end{array}\right] .
$$

Proof. By omitting subscript $i$, the coordinates of a generic point $P$ of link $\mathrm{L}$ are (see Fig. 7)

$$
\begin{aligned}
& x_{p}=x+\xi \cos \alpha-\eta \sin \alpha \\
& y_{p}=y+\xi \sin \alpha+\eta \cos \alpha,
\end{aligned}
$$

from which it is easy to prove that the square of the velocity of $P$ is

$$
\begin{aligned}
& V_{p}^{2}=\dot{x}_{p}^{2}+\dot{y}_{p}^{2}=\dot{x}^{2}+\dot{y}^{2}+\dot{\alpha}^{2}\left(\xi^{2}+\eta^{2}\right)-2 \dot{x} \dot{\alpha}(\xi \sin \alpha+\eta \cos \alpha)+2 \dot{y} \dot{\alpha}(\xi \cos \alpha-\eta \sin \alpha) \\
& =V_{\mathrm{C}^{-}}^{2}+\dot{\alpha}^{2} d^{2}-2 \dot{x} \dot{\alpha}(\xi \sin \alpha+\eta \cos \alpha)+2 \dot{y} \dot{\alpha}(\xi \cos \alpha-\eta \sin \alpha) .
\end{aligned}
$$




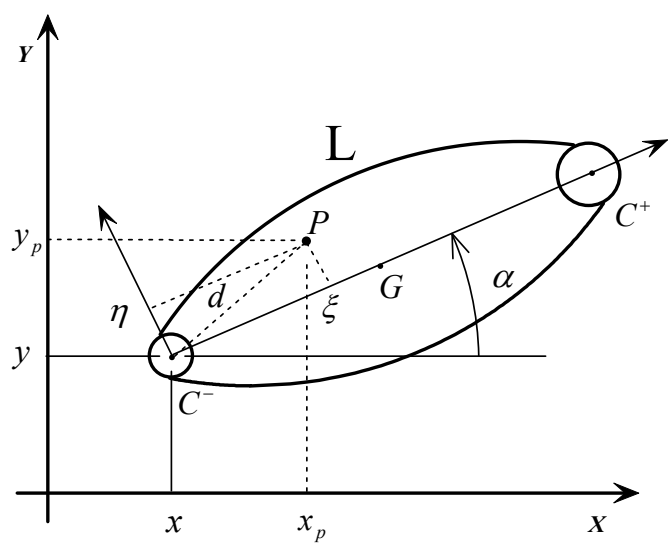

Fig. 7. Determination of the coordinates of a generic point $P$ of the link L.

Since the kinetic energy of link $L$ is $T=\frac{1}{2} \int_{L} V_{p}^{2} d m$, and for the hypothesis made about the characteristics of the link, the proof of the theorem easily follows.

Consider a planar robot with $n$ links (see Fig. 6). The following theorem holds:

Theorem 3 . The kinetic energy of the $i$-th link of a generic planar robot can be calculated by the relationship

$$
T_{i}=\frac{1}{2} \dot{\alpha}_{12 \ldots i}^{T} A_{i} \dot{\alpha}_{12 \ldots i},
$$

where the inertia matrix in terms of the angular coordinates $\alpha_{1 \ldots i}$, expressed by $A_{i}=\left\{a_{i, h k}\right\}$, can be calculated by using the formula:

$$
A_{i}=R_{i}^{T} I_{i} R_{i}
$$

in which

$$
R_{i}=\left[\begin{array}{ccccc}
-L_{1} \sin \alpha_{1} & -L_{2} \sin \alpha_{2} & \cdot & -L_{i-1} \sin \alpha_{i-1} & 0 \\
L_{1} \cos \alpha_{1} & L_{2} \cos \alpha_{2} & . & L_{i-1} \cos \alpha_{i-1} & 0 \\
0 & 0 & . & 0 & 1
\end{array}\right]
$$

or more directly by the formulae:

$$
\begin{gathered}
a_{i, h h}= \begin{cases}M_{i} L_{h}^{2}, & \text { if } h<i \\
J_{i}=M_{i} \rho_{i}^{2}, & \text { if } h=i,\end{cases} \\
a_{i, h k}=\left\{\begin{array}{l}
M_{i} L_{h} L_{k} \cos \left(\alpha_{k}-\alpha_{h}\right), \text { if } h<k<i \\
M_{i} L_{h} L_{G i} \cos \left(\alpha_{i}-\alpha_{h}\right), \text { if } h<k=i .
\end{array}\right.
\end{gathered}
$$

An alternative equivalent and more mnemonic expression for (17a)-(17b) is 
1) $\forall h \leq i ，$

$$
a_{i, h h}=M_{i} \tilde{L}_{h}^{2}, \tilde{L}_{h}=\left\{\begin{array}{l}
L_{h}, \text { if } h<i \\
\rho_{i}, \text { if } h=i
\end{array}\right.
$$

2) $\forall h<k \leq i$,

$$
a_{i, h k}=M_{i} \tilde{L}_{h} \tilde{L}_{k} \cos \left(\alpha_{k}-\alpha_{h}\right), \tilde{L}_{h, k}= \begin{cases}L_{h, k}, & \text { if } h, k<i \\ L_{G i}, & \text { if } h, k=i .\end{cases}
$$

Proof. For the generic $i$-th link, in the hypothesis that $x_{1}=y_{1}=0$, it is

$$
\begin{gathered}
x_{i}=L_{1} \cos \alpha_{1}+L_{2} \cos \alpha_{2}+\ldots+L_{i} \cos \alpha_{i} \\
y_{i}=L_{1} \sin \alpha_{1}+L_{2} \sin \alpha_{2}+\ldots+L_{i} \sin \alpha_{i} \\
\alpha_{i}=\alpha_{i}
\end{gathered}
$$

from which

$$
\begin{gathered}
\dot{x}_{i}=-\dot{\alpha}_{1} L_{1} \sin \alpha_{1}-\dot{\alpha}_{2} L_{2} \sin \alpha_{2}-\ldots-\dot{\alpha}_{i} L_{i} \sin \alpha_{i} \\
\dot{y}_{i}=\dot{\alpha}_{1} L_{1} \cos \alpha_{1}+\dot{\alpha}_{2} L_{2} \cos \alpha_{2}+\ldots+\dot{\alpha}_{i} L_{i} \cos \alpha_{i} \\
\dot{\alpha}_{i}=\dot{\alpha}_{i}
\end{gathered}
$$

or

$$
\dot{q}_{i}=R_{i} \dot{\alpha}_{1 \ldots i}
$$

It follows that

$$
T_{i}=\frac{1}{2} \dot{q}_{i}^{T} I_{i} \dot{q}_{i}=\frac{1}{2} \dot{\alpha}_{12 \ldots i}^{T} R_{i}^{T} I_{i} R_{i} \dot{\alpha}_{1 \ldots i}
$$

and hence (15).

From (15) it is:

1) $\forall h \leq k<i$

$$
a_{i, h k}=\left[\begin{array}{lll}
-L_{h} \sin \alpha_{h} & L_{h} \cos \alpha_{h} & 0
\end{array}\right] \cdot\left[\begin{array}{ccc}
M_{i} & 0 & -N_{i} \sin \alpha_{i} \\
0 & M_{i} & N_{i} \cos \alpha \\
-N_{i} \sin \alpha_{i} & N_{i} \cos \alpha_{i} & J_{i}
\end{array}\right] \cdot\left[\begin{array}{c}
-L_{k} \sin \alpha_{k} \\
L_{k} \cos \alpha_{k} \\
0
\end{array}\right]=M_{i} L_{h} L_{k} \cos \left(\alpha_{k}-\alpha_{h}\right) ;
$$

2) $\forall h<k=i$

$$
a_{i, h i}=\left[\begin{array}{ll}
-L_{h} \sin \alpha_{h} & L_{h} \cos \alpha_{h}
\end{array}\right] \cdot\left[\begin{array}{ccc}
M_{i} & 0 & -N_{i} \sin \alpha_{i} \\
0 & M_{i} & N_{i} \cos \alpha \\
-N_{i} \sin \alpha_{i} & N_{i} \cos \alpha_{i} & J_{i}
\end{array}\right] \cdot\left[\begin{array}{l}
0 \\
0 \\
1
\end{array}\right]=L_{h} N_{i} \cos \left(\alpha_{i}-\alpha_{h}\right)
$$

3) $h=k=i$ 


$$
a_{i, i i}=\left[\begin{array}{lll}
0 & 0 & 1
\end{array}\right] \cdot\left[\begin{array}{ccc}
M_{i} & 0 & -N_{i} \sin \alpha_{i} \\
0 & M_{i} & N_{i} \cos \alpha \\
-N_{i} \sin \alpha_{i} & N_{i} \cos \alpha_{i} & J_{i}
\end{array}\right] \cdot\left[\begin{array}{l}
0 \\
0 \\
1
\end{array}\right]=J_{i}
$$

and hence (17a), (17b).

In view of Theorem 3, the kinetic energy of the robot is

$$
T=T_{1}+T_{2}+\ldots+T_{n}=\frac{1}{2} \dot{\alpha}^{T} A \dot{\alpha},
$$

where

$$
\alpha=\alpha_{1 \ldots n}=\left[\begin{array}{llll}
\alpha_{1} & \alpha_{2} & \ldots & \alpha_{n}
\end{array}\right]^{T},
$$

and the inertia matrix $A=A(\alpha)$ of the robot in terms of the angular coordinates $\alpha$ is obtained by assembling the matrices $A_{i}$ as illustrated in Fig. 8 (namely, by summing to all the principal minors of $A_{n}$ of order $i<n$ the respectie $A_{i}^{\prime}$ s).

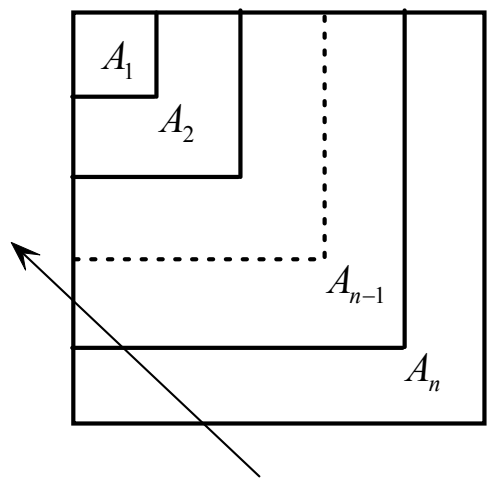

Fig. 8. Graphical representation of the algorithm that computes the robot inertia matrix. In MATLAB-like instructions the algorithm to calculate the inertia matrix is:

$\mathrm{A}=\mathrm{An}$;

for $\mathrm{i}=\mathrm{n}-1:-1: 1$

$\mathrm{A}(1: \mathrm{i}, 1: \mathrm{i})=\mathrm{A}(1: \mathrm{i}, 1: \mathrm{i})+\mathrm{Ai}$;

end.

Moreover, since $\alpha=U \beta$, where

$$
U=\left[\begin{array}{llll}
1 & 0 & . & 0 \\
1 & 1 & . & 0 \\
\cdot & . & . & . \\
1 & 1 & 1 & 1
\end{array}\right],
$$

the kinetic energy of the robot can be calculated by using the relationship alternatively 


$$
T=\frac{1}{2} \dot{\beta}^{T} B \dot{\beta}
$$

where the inertia matrix $B=B(\beta)$ of the robot in terms of the angular coordinates $\beta$ is obtained by

$$
B=U^{T} A(\alpha) U=U^{T} A\left(U^{-1}(\beta)\right) U .
$$

Note that the computation of the inertia matrix is performed by jointly using the absolute and relative coordinates. This allows simplifying, in a natural manner, the algebraic expression of the analytical model.

Clearly, if for a given $\beta$ and hence for a given $\alpha=U \beta$ the interest is only about the numerical value of $B$, it is possible to numerically compute the matrix $A$ preliminarily (from $\alpha$ by using Theorem 3 and the algorithm illustrated in Fig. 7) and then the matrix $B$ through the relationship $B=U^{T} A U$ or through the following algorithm described in MATLAB-like instructions:

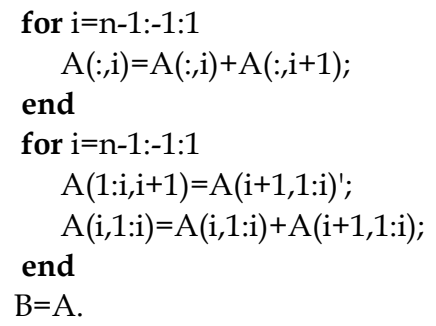

Remark 3. It is important to note that, although by virtue of Theorem 3 the analytical expression of $A_{i}$ is relatively simple, the analytical expression of $A$ is quite complex and, as a result, the expression of $B$ is extremely complex (e.g. the entry $B(1,1)$ of $B$ is the sum of all the elements of all the matrices $A_{i}$ !).

Once the inertia matrix $B$ is obtained, the dynamic model of the robot can be derived by the Euler-Lagrange equation in the usual form (1) or in the alternative form (6). In the case of a planar robot, with link characteristics as required by Theorem 2, the efficiency of the method illustrated in Theorem 1 can be further improved by using the following result.

Theorem 4. The gradient of the kinetic energy of the $i$-th link in terms of $\alpha_{12 \ldots i}\left[\beta_{12 \ldots i}\right]$ can be calculated by the relationship

$$
\frac{\partial T_{i}}{\partial \beta_{12 \ldots i}}=c_{i}=-\left[\begin{array}{c}
0 \\
M_{i} \sum_{h<k: \operatorname{var} \beta_{2}} \widetilde{L}_{h} \dot{\alpha}_{h} \widetilde{L}_{k} \dot{\alpha}_{k} \sin \left(\alpha_{k}-\alpha_{h}\right) \\
M_{i} \sum_{h<k: \operatorname{var} \beta_{3}} \widetilde{L}_{h} \dot{\alpha}_{h} \widetilde{L}_{k} \dot{\alpha}_{k} \sin \left(\alpha_{k}-\alpha_{h}\right) \\
M_{i} \sum_{h<k: \operatorname{var} \beta_{i}} \widetilde{L}_{h} \dot{\alpha}_{h} \widetilde{L}_{k} \dot{\alpha}_{k} \sin \left(\alpha_{k}-\alpha_{h}\right)
\end{array}\right],
$$




$$
\left[\frac{\partial T_{i}}{\partial \beta_{12 \ldots i}}=c_{i}=-\left[\begin{array}{c}
M_{i} \sum_{h<k: \operatorname{var} \beta_{2}} \widetilde{L}_{h} \sum_{p=1}^{h} \dot{\beta}_{p} \widetilde{L}_{k} \sum_{q=1}^{k} \dot{\beta}_{q} \sin \left(\sum_{r=h+1}^{k} \beta_{r}\right) \\
M_{i} \sum_{h<k: \operatorname{var} \beta_{3}} \widetilde{L}_{h} \sum_{p=1}^{h} \dot{\beta}_{p} \widetilde{L}_{k} \sum_{q=1}^{k} \dot{\beta}_{q} \sin \left(\sum_{r=h+1}^{k} \beta_{r}\right) \\
M_{i} \sum_{h<k: \operatorname{var} \beta_{i}} \widetilde{L}_{h} \sum_{p=1}^{h} \dot{\beta}_{p} \tilde{L}_{k} \sum_{q=1}^{k} \dot{\beta}_{q} \sin \left(\sum_{r=h+1}^{k} \beta_{r}\right)
\end{array}\right]\right],
$$

where

$$
\tilde{L}_{m}=\left\{\begin{array}{ll}
L_{m}, & \text { if } m<i \\
L_{G i}, & \text { if } m=i
\end{array}, \quad m=\{h, k\},\right.
$$

and the notation $h<k: \operatorname{var} \beta_{l}, l=2, \ldots, i$, indicates the set of the pairs $h, k$, with $h<k$, such that the angle $\alpha_{h k}=\alpha_{k}-\alpha_{h}=\beta_{h+1}+\beta_{h+2}+\ldots+\beta_{k}$ between the links $h$ and $k$ varies with $\beta_{l}$.

Proof. In view of Theorem 3 , the kinetic energy of the generic $i$-th link is

$$
\begin{gathered}
T_{i}=\frac{1}{2} M_{i} \sum_{h=1, \ldots, i-1} L_{h}^{2} \dot{\alpha}_{h}^{2}+\frac{1}{2} M_{i} \dot{\alpha}_{i}^{2}+M_{i} \sum_{\substack{h=1, \ldots i 1 \\
k=h+1, \ldots, i}} \widetilde{L}_{h} \widetilde{L}_{k} \dot{\alpha}_{h} \dot{\alpha}_{k} \cos \left(\alpha_{k}-\alpha_{h}\right) \\
=T_{c}+M_{i} \sum_{\substack{h=1, \ldots i-1, k=h+1, \ldots, i}} \widetilde{L}_{h} \widetilde{L}_{k} \dot{\alpha}_{h} \dot{\alpha}_{k} \cos \left(\alpha_{k}-\alpha_{h}\right),
\end{gathered}
$$

where $T_{c}$ is the portion of kinetic energy that is independent of $\alpha_{12 \ldots i}$.

From (32) the expression (31a) of the gradient of $T_{i}$ in terms of $\alpha_{12 \ldots i}\left[\beta_{12 \ldots i}\right]$ easily follow.

The vector $c$ can be simply obtained by suitably assembling the various vectors $c_{i}$ (i.e. by progressively summing to the first $i$ components of $c_{n}, i<n$, the respective $c_{i}^{\prime}$ s).

In MATLAB-like instructions:

$\mathrm{c}=\mathrm{cn}$;

for $\mathrm{i}=\mathrm{n}-1:-1: 2$

$c(1: i)=c(1: i)+c i$;

end.

Finally, it is important to note that if the work plane of the robot is vertical, the gravitational torques can be calculated by using the following result.

Theorem 5. If the work plane of the robot is vertical, the weight of the $i$-th link originates a torque on the $k$-th joint, $k \leq i$ :

$$
\gamma_{k}=M_{i} g \sum_{m=k}^{i} \widetilde{L}_{m} \cos \alpha_{m}
$$

where $g$ is the gravitational acceleration and the lengths $\widetilde{L}_{m}$ are given by (31b). 
Proof. The proof easily follows by considering Fig. 6 .

Remark 4. The proposed method efficiency has been explicitly compared to the Articulated Body approach efficiency in terms of number of flops (in Matlab ${ }^{\mathrm{TM}}$ environment), for executing the same elementary integration step by using the absolute Lagrangian coordinates (see Fig. 9). From this figure it results that, for industrial robots with a limited number of links, the proposed method is more efficient.

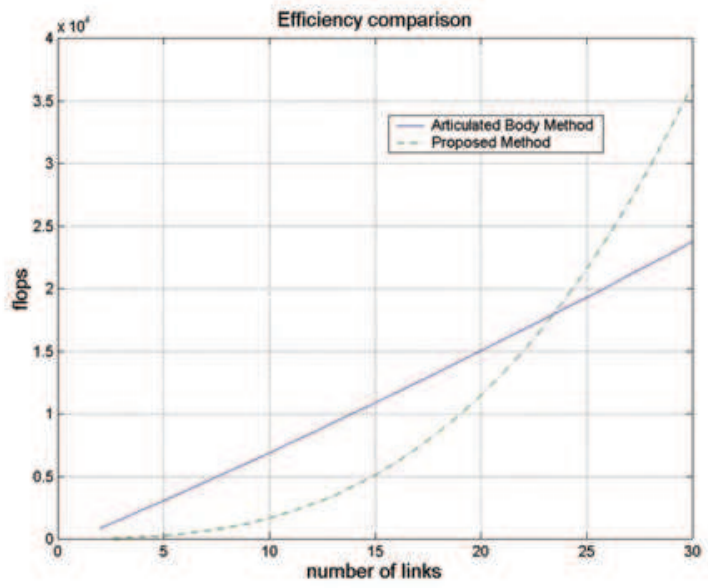

Fig. 9. Efficiency comparison between the Articulated Body method and the proposed method.

Remark 5. The above-derived analytical expressions, in explicit form, of $B, c$ and $\gamma$ are useful for speeding up the simulation, for comparing them to the proposed numerical approach and for finally evaluating which terms can be simplified or neglected in the case of robots with many and/or flexible links. Thus, for instance, for flexible link robots models, obtained via discretization of the links, the terms relative to the deformation angles can be simplified by substituting the sine function with the respective argument or even be completely neglected, considering the terms relative to the motion only.

\section{Spatial robot modeling}

For spatial robots with generic shape links and connected, for the sake of brevity, with spherical joints, several theorems are formulated and demonstrated in a simple manner and some algorithms that allow computing in an efficient way analytically or numerically both the inertia matrix and the gradient of the potential energy are provided.

\subsection{Hypotheses and notations}

In the following, for brevity, only spatial robots constituted by $n$ links of generic shape, connected by spherical joints are considered.

Moreover, the following notations will be used (see Fig. 10): 


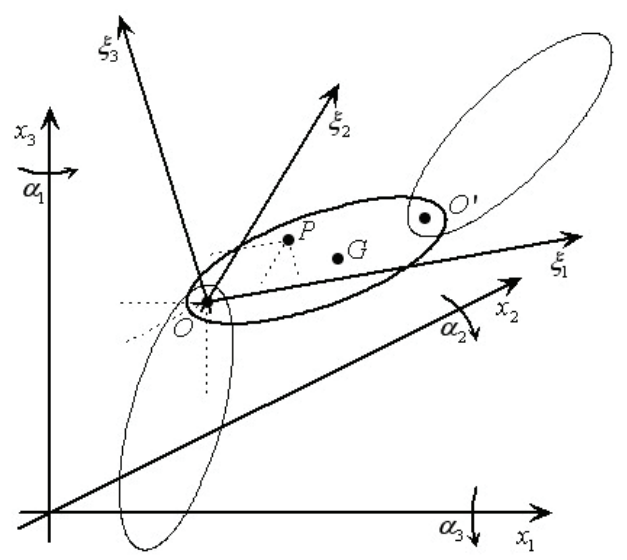

Fig. 10. Coordinates of the generic point $P$ of a link with respect to the body frame and to the inertial frame.

$\mathbf{q}_{i}=\left[\begin{array}{ll}\mathbf{x}_{\mathrm{O} i}^{T} & \mathbf{a}_{i}^{T}\end{array}\right]^{T}=\left[\begin{array}{llllll}x_{\mathrm{O} 1 i} & x_{\mathrm{O} 2 i} & x_{\mathrm{O} i} & \alpha_{1 i} & \alpha_{2 i} & \alpha_{3 i}\end{array}\right]^{T}=$ "absolute coordinates" of the $i$ - th link, $I_{i}=\left\{I_{\text {ihk }}\right\}=$ inertia matrix of the $i$-th link with respect to the axes $\xi_{1}, \xi_{2}, \xi_{3}$,

$\boldsymbol{\xi}_{G i}=\left[\begin{array}{lll}\xi_{G 1 i} & \xi_{G 2 i} & \xi_{G 3 i}\end{array}\right]^{T}=$ coordinates of the center of mass $G_{i}$ in the body frame,

$\xi_{O^{\prime} i}=\left[\begin{array}{lll}\xi_{O^{\prime} 1 i} & \xi_{O^{\prime} 2 i} & \xi_{O^{\prime} 3 i}\end{array}\right]^{T}=$ coordinates of the rotation center $O_{i}^{\prime}$ in the body frame,

$M_{i}=$ total mass of the $i$-th link,

$\mathbf{a}_{1 \ldots i}=\left[\begin{array}{llll}\mathbf{a}_{1}^{T} & \mathbf{a}_{2}^{T} & \ldots & \mathbf{a}_{i}^{T}\end{array}\right]^{T}=$ "angular absolute coordinates" of the chain constituted by the first $i$ links, $\mathfrak{R}_{i}\left(\mathbf{a}_{i}\right)=$ rotation matrix of the $i$-th link,

$R_{i h}=\frac{\partial \Re_{i}\left(\mathbf{a}_{i}\right)}{\partial \alpha_{i h}}$,

$A_{i}=$ inertia matrix of the $i$-th link with respect to the coordinates $\boldsymbol{\alpha}_{1 \ldots i}$,

$\mathbf{a}=\mathbf{a}_{1 \ldots n}=$ "absolute angular coordinates" of the robot,

$A=$ inertia matrix of the robot with respect to the coordinates $\boldsymbol{\alpha}$,

$I=$ identity matrix of order 3,

$s_{h i}=\sin \left(\alpha_{h i}\right)$,

$c_{h i}=\cos \left(\alpha_{h i}\right)$.

\subsection{Main results}

Let us consider a generic link of a generic shape of a spatial robot with spherical joints.

The following theorems and algorithms for the computation of the kinetic energy $T_{i}$ of the $i$ th link first as a function of the absolute coordinates $\mathbf{q}_{i}$ of the $i$-th link and then as a function of the absolute angular coordinates $\mathbf{a}_{1 \ldots i}$ of the chain constituted by the first $i$ links are provided.

Theorem 6. The kinetic energy of the $i$-th link may be calculated by means of the relationship 


$$
T_{i}=\frac{1}{2} \dot{\mathbf{q}}_{i}^{T} \mathfrak{\Im}_{i} \dot{\mathbf{q}}_{i}
$$

where

$$
\mathfrak{I}_{i}=\left[\begin{array}{cc}
M_{i} I & M_{i} \sum_{h} R_{h i} \xi_{G h i} \\
M_{i} \sum_{h} R_{h i}^{T} \xi_{G h i} & \sum_{h, k} R_{h i}^{T} R_{k i} I_{h k i}
\end{array}\right] .
$$

Proof. By omitting subscript $i$, the coordinates of the generic point $P$ of the link in the fixed frame (see Fig. 10) are given by

$$
\mathbf{x}_{P}=\mathbf{x}_{O}+\mathfrak{R}(\mathbf{\alpha}) \boldsymbol{\zeta}_{P},
$$

where $\mathfrak{R}$ is the rotation matrix of the body frame with respect to the fixed frame and $\xi_{P}$ is the vector of the coordinates of the generic point $P$ of the link in the body frame.

From (36) it follows

$$
\dot{\mathbf{x}}_{P}=\dot{\mathbf{x}}_{O}+\sum_{h} R_{h} \xi_{P h} \dot{\mathbf{\alpha}}
$$

where

$$
R_{h}=\frac{\partial \Re}{\partial \alpha_{h}}, h=1,2,3 .
$$

From (37) it is

$$
V^{2}=\dot{\mathbf{x}}_{P}^{T} \dot{\mathbf{x}}_{P}=\dot{\mathbf{x}}_{0}^{T} \dot{\mathbf{x}}_{0}+\dot{\mathbf{\alpha}}^{T} \sum_{h, k} R_{h}^{T} R_{k} \xi_{P h} \xi_{P k} \dot{\mathbf{\alpha}}+2 \dot{\mathbf{x}}_{0}^{T} \sum_{h} R_{h} \xi_{P h} \dot{\mathbf{\alpha}}
$$

from which

$$
\begin{aligned}
& T=\frac{1}{2} \int V^{2} d m= \\
& =\frac{1}{2} M \dot{\mathbf{x}}_{0}^{T} \dot{\mathbf{x}}_{0}+\frac{1}{2} \dot{\mathbf{\alpha}}^{T} \sum_{h, k} R_{h}^{T} R_{k} I_{h k} \dot{\mathbf{\alpha}}+M \dot{\mathbf{x}}_{0}^{T} \sum_{h} R_{h} \xi_{G h} \dot{\mathbf{\alpha}}= \\
& =\frac{1}{2}\left(\begin{array}{ll}
\dot{\mathbf{x}}_{0}^{\mathrm{T}} & \dot{\mathbf{\alpha}}^{\mathrm{T}}
\end{array}\right)\left[\begin{array}{cc}
M I & M \sum_{h} R_{h} \xi_{G h} \\
M \sum_{h} R_{h}^{T} \xi_{G h} & \sum_{h, k} R_{h}^{T} R_{k} I_{h k}
\end{array}\right]\left(\begin{array}{c}
\dot{\mathbf{x}}_{0} \\
\dot{\mathbf{\alpha}}
\end{array}\right)
\end{aligned}
$$

and hence the proof.

Remark 6. The vector of the coordinates of the center of mass $\xi_{G_{i}}$ and the inertia matrix $\left\{I_{h k}\right\}$ of the $i$-th link, in the case of a complex structure, may be easily evaluated by making use of software packages, such as CATIATM.

Theorem 7. If the Euler angles of the $i$-th link $\boldsymbol{\alpha}_{i}=\left[\begin{array}{llll}\boldsymbol{\alpha}_{1 i} & \boldsymbol{\alpha}_{2 i} & \boldsymbol{\alpha}_{3 i}\end{array}\right]^{T}$ are the ZYX angles, also called RPY angles (Roll-Pitch-Yaw), (see Fig. 10), by omitting subscript $i$, the matrices $R_{h}$ result: 


$$
\begin{gathered}
R_{1}=\left[\begin{array}{ccc}
-s_{1} c_{2} & -s_{1} s_{2} s_{3}-c_{1} c_{3} & -s_{1} s_{2} c_{3}+c_{1} s_{3} \\
c_{1} c_{2} & c_{1} s_{2} s_{3}-s_{1} c_{3} & c_{1} s_{2} c_{3}+s_{1} s_{3} \\
0 & 0 & 0
\end{array}\right] \\
R_{2}=\left[\begin{array}{ccc}
-c_{1} s_{2} & c_{1} c_{2} s_{3} & c_{1} c_{2} c_{3} \\
-s_{1} s_{2} & s_{1} c_{2} s_{3} & s_{1} c_{2} c_{3} \\
-c_{2} & -s_{2} s_{3} & -s_{2} c_{3}
\end{array}\right] \\
R_{3}=\left[\begin{array}{ccc}
0 & c_{1} s_{2} c_{3}+s_{1} s_{3} & -c_{1} s_{2} s_{3}+s_{1} c_{3} \\
0 & s_{1} s_{2} c_{3}-c_{1} s_{3} & -s_{1} s_{2} s_{3}-c_{1} c_{3} \\
0 & c_{2} c_{3} & -c_{2} s_{3}
\end{array}\right]
\end{gathered}
$$

Proof. The rotation matrix $\mathfrak{R}_{i}\left(\mathbf{a}_{i}\right)$ of the $i$-th link, by omitting subscript $i$, results (43)

$$
\Re(\mathbf{\alpha})=\left[\begin{array}{ccc}
c_{1} c_{2} & c_{1} s_{2} s_{3}-s_{1} c_{3} & c_{1} s_{2} c_{3}+s_{1} s_{3} \\
s_{1} c_{2} & s_{1} s_{2} s_{3}+c_{1} c_{3} & s_{1} s_{2} c_{3}-c_{1} s_{3} \\
-S_{2} & c_{2} s_{3} & c_{2} c_{3}
\end{array}\right],
$$

from which, for (38), the proof.

The computation of the matrices $\mathfrak{I}_{i}$ may be sped up by the following algorithm, where subscript $i$ has been omitted.

\section{Algorithm 1.}

Step 1. Compute

$$
s_{1}, s_{2}, s_{3} ; c_{1}, c_{2}, c_{3}
$$

Step 2. Compute

$$
\begin{array}{ll}
s_{1} s_{2}, s_{1} s_{3}, s_{2} s_{3} ; & c_{1} c_{2}, c_{1} c_{3}, c_{2} c_{3} ; \\
s_{1} c_{2}, s_{1} c_{3}, s_{2} c_{3} ; & c_{1} s_{2}, c_{1} s_{3}, c_{2} s_{3} ; \\
\left(s_{1} s_{2}\right) s_{3},\left(s_{1} s_{2}\right) c_{3} ; & \left(s_{1} c_{2}\right) s_{3},\left(s_{1} c_{2}\right) c_{3} ; \\
\left(c_{1} c_{2}\right) s_{3},\left(c_{1} c_{2}\right) c_{3} ; & \left(c_{1} s_{2}\right) s_{3},\left(c_{1} s_{2}\right) c_{3} .
\end{array}
$$

Step 3.Compute

$$
R_{1}^{T} R_{1}, R_{1}^{T} R_{2}, R_{1}^{T} R_{3} ; \quad R_{2}^{T} R_{2}, R_{2}^{T} R_{3} ; \quad R_{3}^{T} R_{3}
$$

If the axis $\xi_{1 i}$ of the body frame is chosen such as to contain the center of gravity $G_{i}$ and it results principal of inertia then, by choosing the other two axes parallel to the other inertia principal axes, the computation of the matrix $\mathfrak{I}_{i}$ may be further sped up, as it results from the following theorem.

Theorem 8. If the body reference axes of the $i$-th link are parallel to the inertia principal axes and the center of gravity $G_{i}$ belong to the axis $\xi_{1 i}$, the matrix $\mathfrak{I}_{i}$ results 


$$
\mathfrak{J}_{i}=\left[\begin{array}{cc}
M_{i} I & M_{i} R_{1 i} \xi_{G 1 i} \\
M_{i} R_{1 i}^{T} \xi_{G 1 i} & \sum_{h} R_{h i}^{T} R_{h i} I_{h h i}
\end{array}\right] .
$$

Proof. The proof easily follows from (35) and from

$$
\xi_{G 2 i}=0, i=2,3 ; \quad I_{h k i}=0, h=1,2, k=h+1,3 .
$$

Let us consider now a robot with $n$ links (see Fig. 11). The following theorems and algorithms for the computation of the inertia matrix $A$ of the whole robot, as a function of the absolute angular coordinates a of the robot, hold.

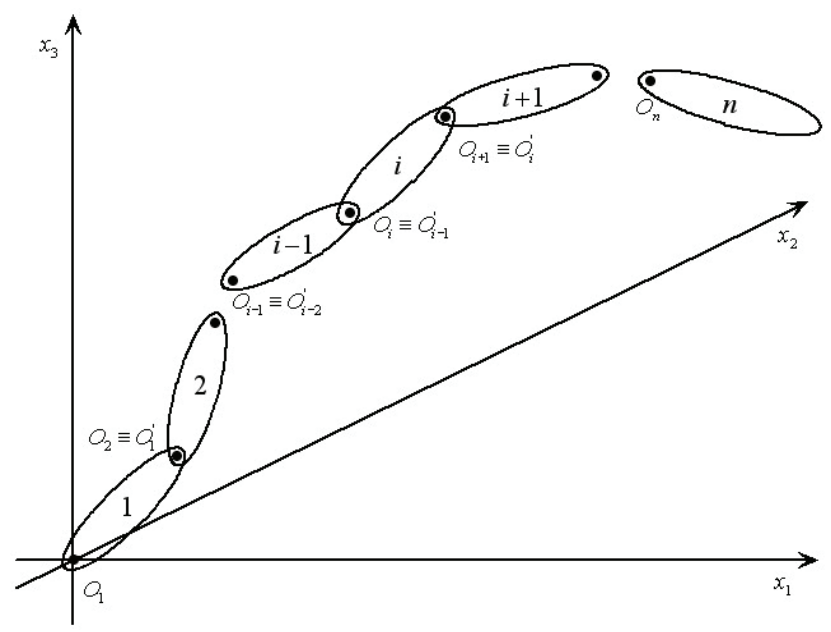

Fig. 11. Schematic representation of a spatial robot with $n$ links.

Theorem 9. The kinetic energy of the $i$-th link of a generic spatial robot may be computed by means of the relationship

$$
T_{i}=\frac{1}{2} \dot{\mathbf{a}}_{1 \ldots i}^{\mathbf{T}} A_{i} \dot{\mathbf{a}}_{1 \ldots i}
$$

where

$$
A_{i}=\left\{\begin{array}{c}
\mathfrak{I}_{221}, \text { if } i=1 \\
{\left[\begin{array}{cc}
M_{i} V_{1 \ldots i-1}{ }^{T} V_{1 \ldots i-1} & V_{1 \ldots i-1}{ }^{T} \mathfrak{I}_{12 i} \\
\mathfrak{J}_{12 i}^{T} V_{1 \ldots i-1} & \mathfrak{I}_{22 i}
\end{array}\right], \quad \text { if } i=2,3, \ldots, n}
\end{array}\right.
$$

in which:

$$
\begin{gathered}
\mathfrak{J}_{12 i}=M_{i} \sum_{h} R_{h i} \xi_{G h i}, \quad \mathfrak{I}_{22 i}=\sum_{h, k} R_{h i}^{T} R_{k i} I_{h k i} \\
V_{1 \ldots i-1}=\left[\begin{array}{llll}
V_{1} & V_{2} & \cdots & V_{i-1}
\end{array}\right], \quad V_{j}=\sum_{h} R_{h j} \xi_{O^{\prime} h j}, j=1,2, \ldots, i-1 .
\end{gathered}
$$


Proof. From the following relationships (see Fig. 11 and (37))

$$
\dot{\mathbf{x}}_{O i}=\dot{\mathbf{x}}_{O^{\prime} i-1}=\dot{\mathbf{x}}_{O i-1}+\sum_{h} R_{h i-1} \xi_{O^{\prime} h i-1} \dot{\mathbf{a}}_{i-1}, \quad i=2, \ldots, n, \quad \dot{\mathbf{x}}_{O 1}=0,
$$

it is

$$
\dot{\mathbf{x}}_{O i}=\left[\begin{array}{llll}
V_{1} & V_{2} & \cdots & V_{i-1}
\end{array}\right]\left[\begin{array}{c}
\dot{\mathbf{q}}_{1} \\
\dot{\mathbf{\alpha}}_{2} \\
\vdots \\
\dot{\mathbf{a}}_{i-1}
\end{array}\right]=V_{1 \ldots i-1} \dot{\mathbf{a}}_{1 \ldots i-1},
$$

from which

$$
\dot{\mathbf{q}}_{i}=\left[\begin{array}{c}
\dot{\mathbf{x}}_{O i} \\
\dot{\mathbf{\alpha}}_{i}
\end{array}\right]=\left[\begin{array}{c}
V_{1 \ldots i-1} \dot{\mathbf{\alpha}}_{1 \ldots i-1} \\
\dot{\mathbf{\alpha}}_{i}
\end{array}\right]=\left[\begin{array}{cc}
V_{1 \ldots i-1} & 0 \\
0 & I
\end{array}\right] \dot{\mathbf{\alpha}}_{1 \ldots i} .
$$

By substituting (55) in (34), it is

$$
\begin{aligned}
& T_{i}=\frac{1}{2} \dot{\mathbf{q}}_{i}^{T} \mathfrak{J}_{i} \dot{\mathbf{q}}_{i}= \\
& =\frac{1}{2} \dot{\mathbf{a}}_{1 \ldots i}^{T}\left[\begin{array}{cc}
V_{1 \ldots i-1}{ }^{T} & 0 \\
0 & I
\end{array}\right]\left[\begin{array}{cc}
M_{i} I & \mathfrak{I}_{12 i} \\
\mathfrak{J}^{T} & \mathfrak{I}_{22 i}
\end{array}\right]\left[\begin{array}{cc}
V_{1 \ldots i-1} & 0 \\
0 & I
\end{array}\right] \dot{\mathbf{\alpha}}_{1 \ldots i}= \\
& =\frac{1}{2} \dot{\mathbf{\alpha}}_{1 \ldots i}^{T}\left[\begin{array}{cc}
M_{i} V_{1 \ldots i-1}{ }^{T} V_{1 \ldots i-1} & V_{1 \ldots i-1}{ }^{T} \mathfrak{I}_{12 i} \\
\mathfrak{J}_{12 i}^{T} V_{1 \ldots i-1} & \mathfrak{J}_{22 i}
\end{array}\right] \dot{\mathbf{\alpha}}_{1 \ldots i}
\end{aligned}
$$

from which the proof follows.

The next theorem, similar to Theorem 8 , is useful to simplify the computation of the matrices $A_{i}$.

Theorem 10. If the axes of the body frame of link $j, j=1,2, \ldots, i-1$, are parallel to the inertia principal axes and the centers of gravity $G_{j}$ and the rotation centers $O_{j}^{\prime}$ belong to the axis $\xi_{1 j}$, matrices (52) may be more easily calculated by the relations:

$$
\begin{gathered}
\mathfrak{I}_{12 i}=M_{i} R_{1 i} \xi_{G 1 i}, \quad \mathfrak{I}_{22 i}=\sum_{h} R_{h i}^{T} R_{h i} I_{h h i}, \\
V_{1 \ldots i-1}=\left[\begin{array}{llll}
V_{1} & V_{2} & \cdots & V_{i-1}
\end{array}\right], \quad V_{j}=R_{1 j} \xi_{O^{\prime} 1 j}, \quad j=1,2, \ldots, i-1 .
\end{gathered}
$$

Proof. The proof easily follows from Theorem 8 and from the fact that

$$
\xi_{O^{\prime} h j}=0, \quad h=2,3, j=1,2, \ldots, i-1 .
$$

The following theorems may be effectively used to compute the gradient of the gravitational potential energy.

Theorem 11. The gradient of the gravitational potential energy of the $i$-th link $U_{i}$ with respect to the angular coordinates $\mathbf{a}_{1 \ldots i}$ may be calculated by the formula 


$$
\begin{aligned}
& \frac{\partial U_{i}}{\partial \mathbf{a}_{1 \ldots i}}=\mathbf{\gamma}_{i}= \\
& -M_{i}\left[R_{11} \xi_{0_{1}^{\prime}} R_{21} \xi_{0_{1}^{\prime}} R_{31} \xi_{0_{1}^{\prime}} R_{12} \xi_{0_{2}^{\prime}} R_{22} \xi_{0_{2}^{\prime}} R_{32} \xi_{0_{2}^{\prime}} \cdots\right. \\
& \left.\quad \cdots R_{1 i} \xi_{G_{i}} R_{2 i} \xi_{G_{i}} R_{3 i} \xi_{G_{i}}\right]^{T} \mathbf{g}
\end{aligned}
$$

where $\mathbf{g}$ is the gravity acceleration vector in the inertial frame.

Proof. It is easy to verify that the gravitational potential energy of the generic $i$-th link results

$$
U_{i}=-M_{i} \mathbf{g}^{T}\left(\Re_{1}\left(\mathbf{\alpha}_{1}\right) \mathcal{\zeta}_{O_{1}^{\prime}}+\Re_{2}\left(\mathbf{\alpha}_{2}\right) \mathcal{\zeta}_{O_{2}^{\prime}}+\cdots+\Re_{i}\left(\mathbf{\alpha}_{i}\right) \mathcal{\xi}_{G_{i}}\right) .
$$

Relation (60) easily follows from (61).

The next theorem is useful to simplify the computation of $\frac{\partial U_{i}}{\partial \mathbf{a}_{1 \ldots i}}$.

Theorem 12. If the $x_{3}$-axis of the inertial frame is vertical and upwards oriented, the rotation centers $O_{j}^{\prime}$ of the $j$-th links, $j=1,2, \ldots, i-1$, belong to the axes $\xi_{1 j}$ of the body frames, the center of gravity $G_{i}$ of the $i$-th link belongs to the axis $\xi_{1 i}$ and the Euler angles of the $j$-th links, $j=1,2, \ldots, i$, are the $Z Y X$ angles, the gradient $\frac{\partial U_{i}}{\partial \mathbf{a}_{1 \ldots i}}$ may be calculated much more easily by the relationship

$$
\begin{aligned}
& \frac{\partial U_{i}}{\partial \mathbf{a}_{1 \ldots i}}=\mathbf{Y}_{i}= \\
& =M_{i \delta} g\left[\begin{array}{llllllllll}
0 & c_{21} \xi_{O_{1}^{\prime} 1} & 0 & 0 & c_{22} \xi_{O_{2}^{\prime} 1} & 0 & \cdots & 0 & c_{2 i} \xi_{G_{i} 1} & 0
\end{array}\right]^{T} .
\end{aligned}
$$

Proof. Since the $x_{3}$-axis is vertical and upwards oriented it is $\mathbf{g}=\left[\begin{array}{lll}0 & 0 & -g\end{array}\right]^{T}$, where $g$ is the gravity acceleration. Moreover, since $O_{j}^{\prime} \in \xi_{1 j}, j=1,2, \ldots, i-1, G_{i} \in \xi_{1 i}$ :

$$
\xi_{O_{i}^{\prime}}=\left[\begin{array}{lll}
\xi_{O_{i}^{\prime} 1} & 0 & 0
\end{array}\right]^{T}, \xi_{G_{i}}=\left[\begin{array}{lll}
\xi_{G_{i} 1} & 0 & 0
\end{array}\right]^{T}, i=1 \ldots n
$$

The proof easily follows from (41)-(43), (60), (63).

\subsection{Application of the main results}

On the base of Theorem 9 the kinetic energy of the whole robot is

$$
T=T_{1}+T_{2}+\ldots+T_{n}=\frac{1}{2} \dot{\mathbf{\alpha}}^{\mathrm{T}} A \dot{\mathbf{\alpha}},
$$

where the inertia matrix $A=A(\mathbf{\alpha})$ is obtained by assembling the matrices $A_{i}$ according to the following efficient algorithm written in Matlab-like language: 
$\mathrm{A}=\mathrm{An}$;

for $\mathrm{i}=\mathrm{n}-1:-1: 1$

$A\left(1: 3^{*} i, 1: 3 * i\right)=A(1: 3 * i, 1: 3 * i)+A i$;

end.

Once the vectors $\gamma_{i}, i=1,2, \ldots, n$, have been computed by the (60) or the (62), the gradient of the gravity energy $\gamma$ is obtained by assembling the $\gamma_{i}$ according to the following efficient algorithm written in Matlab-like language:

$\gamma=\gamma \mathrm{n}$

for $\mathrm{i}=\mathrm{n}-1:-1: 1$

$\gamma(1: i)=\gamma(1: i)+\gamma i$

end.

Finally, by using the efficient integration scheme shown in Fig. 1 (Celentano \& Iervolino, 2006), it is possible to get the dynamic model of the robot in a simple way.

\subsection{Comparison in terms of efficiency with the articulated-body method}

The efficiency of the method of modeling and simulation proposed for spatial robots has been explicitly compared to the efficiency of a Newtonian method of order $\mathrm{N}$ considered one of the most efficient methods in the literature, i.e. the Articulated-Body Method (Featherstone, 1987), (Featherstone \& Orin, 2000).

More in details, such comparison has been executed in terms of number of flops (in MATLABTM environment), for the execution of the same integration step (see Table 1).

\begin{tabular}{|c|c|c|}
\hline $\begin{array}{c}\text { Number of } \\
\text { links }\end{array}$ & $\begin{array}{c}\text { Flops of the } \\
\text { proposed method }\end{array}$ & $\begin{array}{c}\text { Flops of the } \\
\text { Articulated-Body } \\
\text { method }\end{array}$ \\
\hline 1 & 362 & 1269 \\
\hline 2 & 2459 & 7168 \\
\hline 3 & 8400 & 13758 \\
\hline 4 & 21451 & 20804 \\
\hline 5 & 45868 & 28252 \\
\hline
\end{tabular}

Table 1. Efficiency comparison between the proposed method and the Articulated-Body method.

From Table 1 it results that for practical spatial robots (which of course have a limited number of links), the proposed method is more efficient when $n<4$, although spatial robots with three degrees of freedom spherical joints have been considered. Moreover, it is important to note that the great easiness of the proposed method with respect to the Articulated-Body one is an important index of its efficiency.

However, the authors have the plan to continue and to improve this comparison.

\section{Elements of flexible robots modeling}

\subsection{Methodology}

The results stated in the previous sections allow obtaining, quite simply, accurate and efficient, from a computational point of view, finite-dimensional models.

These models, for rectilinear links, can be obtained approximating the $i$-th link as follows: 
1. in order to compute the kinetic energy and, therefore, the inertia matrix and the gradient of the kinetic energy, with $v_{i}+1$ rigid sublinks with lengths $L_{1 i}, \ldots, L_{v_{i}+1, i}$, interconnected by relative angles $\beta_{1 i}, \ldots, \beta_{v_{i} i}$ (see Fig. 12);

2. in order to compute the elastic energy, with $v_{i}$ flexible sublinks joined and deformable such that their axes make a suitable spline tangent to the axes of the intermediate rigid sublinks in the extreme points of the link and to the intermediate rigid sublinks in suitable interior points (see Fig. 12).

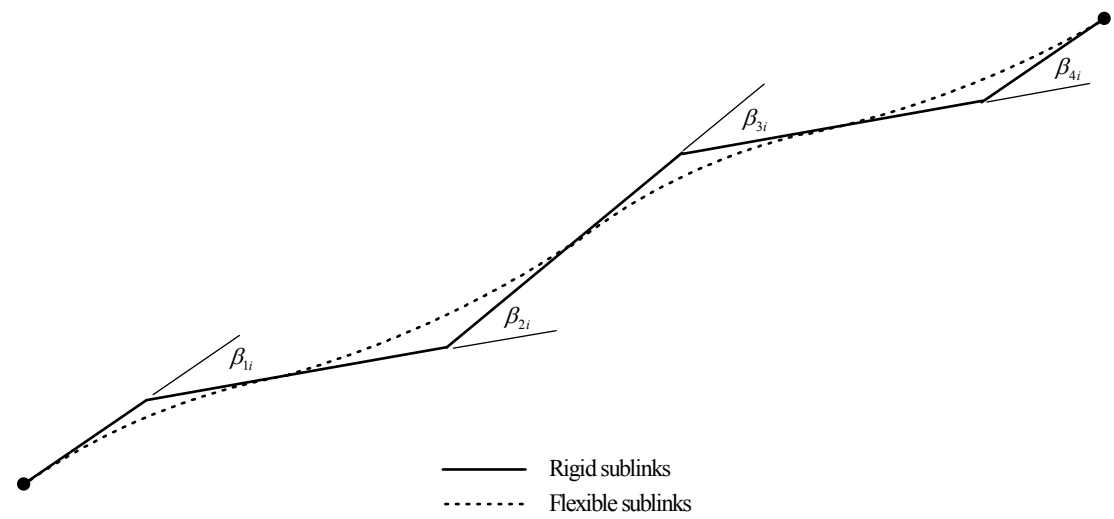

Fig. 12. Approximation of a flexible link with $v_{i}+1=5$ rigid sublinks and with $v_{i}=4$ flexible sublinks congruent to each other at the end.

In this way, neglecting, about the computation of the kinetic energy, the deformation angles respect to the angles of motion, it is easy to verify, from the results of Sections 2 and 3, that the inertia matrix can be computed as follows:

$$
\begin{aligned}
B & =B\left(\beta_{2}, \ldots, \beta_{n}\right)= \\
& =B_{1}+B_{2} \cos \beta_{2}+\ldots+B_{n} \cos \beta_{n}+B_{23} \cos \left(\beta_{2}+\beta_{3}\right)+B_{24} \cos \left(\beta_{2}+\beta_{3}+\beta_{4}\right)+\ldots+B_{n-1, n} \cos \left(\beta_{n-1}+\beta_{n}\right)^{\prime}
\end{aligned}
$$

where $\beta_{i}$ is the angle of motion between the $(i-1)$-th link and the $i$-th link, $i=2, \ldots, n$ (see Fig. 13).

From (65) it is easy to compute also the gradient of the kinetic energy respect to $\beta=\left[\beta_{1} \beta_{11} \ldots \beta_{v_{1} 1} \beta_{2} \beta_{12} \ldots \beta_{v_{2} 2} \beta_{n} \beta_{1 n} \ldots \beta_{v_{n} n}\right]^{T}$ using the following relation

$$
c=\frac{1}{2}\left[\begin{array}{lllllll}
0_{1, v_{1}+1} & \dot{\beta}^{T} \frac{\partial B}{\partial \beta_{2}} \dot{\beta} & \ldots & 0_{1, v_{n-1}} & \dot{\beta}^{T} \frac{\partial B}{\partial \beta_{n}} \dot{\beta} & 0_{1, v_{n}}
\end{array}\right]^{T}
$$

There are different ways of approximating a flexible link using a chain of rigid sublinks and flexible sublinks congruent to each other at the end (Celentano, 2007). 


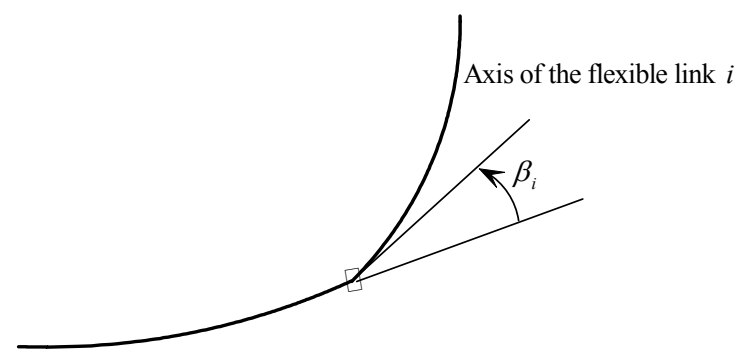

Axis of the flexible link $i-1$

Fig. 13. Angle of motion between two flexible consecutive links.

In the hypothesis that the stiffness of the $i$-th link is uniform, a very efficient way for the choice of the sublinks is to consider the intermediate rigid sublinks with a length of $L_{i} / v_{i}$, the extreme ones with an half length, the flexible sublinks with a length of $L_{i} / v_{i}$ and deformable as parabolic arcs. Consequetly the elastic energy of the $i$-th link results

$$
U_{i}=\frac{1}{2} K_{i}\left(\beta_{1 i}^{2}+\cdots+\beta_{v_{i}}^{2}\right) \text {, }
$$

where

$$
K_{i}=\frac{E_{i} I_{i}}{L_{i} / v_{i}}
$$

is the stiffness of the flexible sublinks.

Therefore the vector of the elastic torques is

$$
\gamma=\left[0 K_{1} \beta_{11} K_{1} \beta_{21} \cdots K_{1} \beta_{v_{1} 1} 0 K_{2} \beta_{12} K_{2} \beta_{22} \cdots K_{2} \beta_{v_{2} 2} \cdots 0 K_{n} \beta_{1 n} K_{n} \beta_{2 n} \cdots K_{n} \beta_{v_{n} n}\right]^{T} .
$$

Remark 7. The proposed method is simple to apply, is very efficient from a computational point of view and it provides a model with null error when the control and disturbance torques are constant. For control actions and disturbances, also in forces, with a fixed and limited band-pass, this model provides an error strongly decreasing when the number of sublinks increase. This fact clearly emerges from the following example.

\subsection{Example: Modeling of a Single-Link Flexible Arm}

Consider a flexible robot with a single flexible link (see Fig. 14).

If the link is a steel bar with a length of $L=2 m$ and with a squared section of side $l=0.01 \mathrm{~m}=1 \mathrm{~cm}$, the first five frequencies computed with the theoretic formulae:

$$
f_{i}=\sqrt{\frac{E I}{m}} \frac{\pi}{32 L^{2}}(4 i+1)^{2}, \quad i=1,2, \cdots, 5
$$

where $E$ is the modulus of elasticity of Joung $\left(\cong 10^{11} \mathrm{~N} / \mathrm{m}^{2}\right), I=l^{4} / 12$ is the moment of inertia of the section respect to the deflection axis and $m \cong 7876 l^{2} \mathrm{Kg} / \mathrm{m}$ is the mass per unit length of the link, are: 


$$
f=6.31,20.4,42.7,73.0,111.3 \mathrm{~Hz} \text {. }
$$

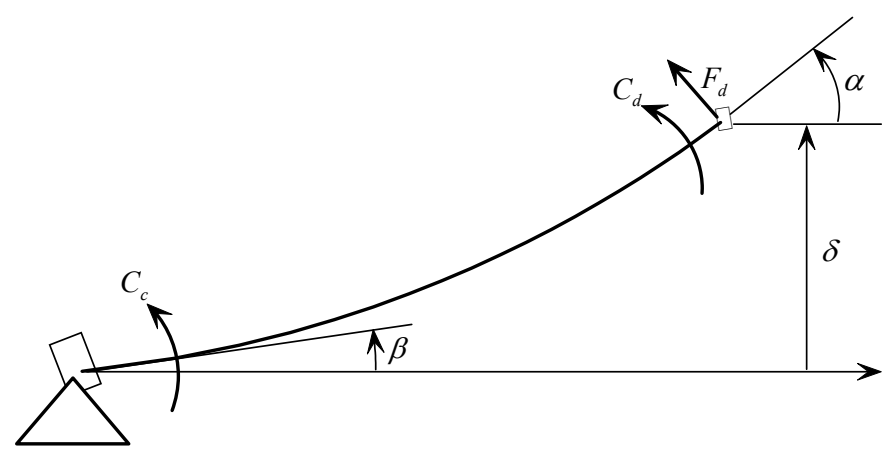

Fig. 14. Schematic representation of a single-link flexible arm.

The frequencies obtained with the proposed method, dividing the link into five and ten flexible sublinks, respectively result:

$$
\begin{gathered}
f=6.31,20.4,41.9,65.6,90.8 \mathrm{~Hz} \\
f=6.31,20.4,42.6,72.77,110.5 \mathrm{~Hz} .
\end{gathered}
$$

Stressing the link with $C_{c}=-1 N m, C_{d}=1 N m, F_{d}=0 N$, if $\beta=0$, the theoretic values of $\alpha$ and $\delta$ are:

$$
\alpha=\frac{L}{E I} C_{d}=1.375^{\circ}, \quad \delta=\frac{L^{2}}{2 E I} C_{d}=2.40 \mathrm{~cm}
$$

It is worth noting that these values and the ones obtained using the proposed method are coincident $\forall v \geq 1$ (Celentano, 2007).

Applying to the link $C_{c}=-2 N m, C_{d}=0 N m, F_{d}=1 N$, if $\beta=0$, the theoretic values of $\alpha$ and $\delta$ result:

$$
\alpha=\frac{L^{2}}{2 E I} F_{d}=1.375^{\circ}, \delta=\frac{L^{3}}{3 E I} F_{d}=3.20 \mathrm{~cm} .
$$

The first value (the orientation angle of payload due to the arm deflection) and the ones computed with the proposed method $\forall v \geq 1$ are coincident, while the second value (the motion of the payload due to the arm deflection) obtained using the proposed method has a relative error of (Celentano, 2007)

$$
\varepsilon_{\delta}=-\frac{1}{4 v^{2}}=\left\{\begin{array}{cl}
-0.01=1 \%, & \text { if } v=5 \\
-0.0025=0.25 \%, & \text { if } v=10 .
\end{array}\right.
$$

\section{Conclusion}

In this chapter an innovative method for robots modeling and simulation, based on an appropriate mathematical formulation of the relative equations of motion and on a new 
integration scheme, has been illustrated. The proposed approach does require the calculation of the inertia matrix and of the gradient of the kinetic energy only. It provides a new analytical-numerical methodology, that has been shown to be simpler and numerically more efficient than the classical approaches, requires no a priori specialized knowledge of the dynamics of mechanical systems and is formulated in order to allow students, researchers and professionals to easily employ it for the analysis of manipulators with the complex-shaped links commonly used in industry.

In the case of planar robots with revolute joints, theorems have been stated and proved that offer a particularly simple and efficient method of computation for both the inertia matrix and the gradient of the kinetic energy. Then a comparison has been made in terms of efficiency between the proposed method and the Articulated-Body one.

Moreover, for spatial robots with generic shape links and connected, for the sake of brevity, with spherical joints, several theorems have been formulated and demonstrated in a simple manner and some algorithms that allow efficiently computing, analytically the inertia matrix, analytically or numerically the gradient of the kinetic and of the gravitational energy have been provided. Furthermore, also in this case a comparison of the proposed method in terms of efficiency with the Articulated-Body one has been reported.

Finally, a methodology for flexible robots modeling, that allow obtaining, quite simply, accurate and efficient, from a computational point of view, finite-dimensional models, has been provided. This method is illustrated with a very significant example.

\section{References}

Celentano, L. (2007). An Innovative and Efficient Method for Flexible Robots Modeling and Simulation. Internal Report, Dipartimento di Informatica e Sistemistica, Università degli Studi di Napoli Federico II, Napoli, Italy, October 2007

Celentano, L. and Iervolino, R. (2007). A Novel Approach for Spatial Robots Modeling and Simulation. MMAR07, 13 th IEEE International Conference on Methods and Models in Automation and Robotics, pp. 1005-1010, Szczecin, Poland, 27-30 August 2007

Celentano, L. and Iervolino, R. (2006). A New Method for Robot Modeling and Simulation. ASME Journal of Dynamic Systems, Measurement and Control, Vol. 128, December 2006, pp. 811-819

Celentano, L. (2006). Modellistica e Controllo dei Sistemi Meccanici Rigidi e Flessibili. PhD Thesis, Dipartimento di Informatica e Sistemistica, Università degli Studi di Napoli Federico II, Napoli, Italy, November 2006

De Wit, C.C., Siciliano, B. and Bastin, G. (1997). Theory of Robot Control, (2nd Ed.) SpringerVerlag, London, UK

Featherstone, R. and Orin, D.E. (2000). Robot Dynamics: Equations and Algorithms. Proceedings of the 2000 IEEE International Conference on Robotics and Automation, pp. 826-834

Featherstone, R. (1987). Robot Dynamics Algorithms, Kluwer Academic Publishers, Boston/ Dordrecht/ Lancaster

Khalil, W. and Dombre, E. (2002). Modelling, Identification and Control of Robots, Hermes Penton Science, London, UK

Sciavicco, L. and Siciliano, B. (2000). Modeling and Control of Robot Manipulators, (2nd Ed.) Springer-Verlag, London, UK 


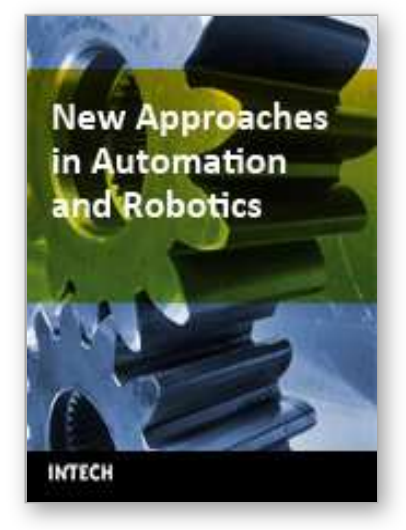

\author{
New Approaches in Automation and Robotics \\ Edited by Harald Aschemann
}

ISBN 978-3-902613-26-4

Hard cover, 392 pages

Publisher I-Tech Education and Publishing

Published online 01, May, 2008

Published in print edition May, 2008

The book New Approaches in Automation and Robotics offers in 22 chapters a collection of recent developments in automation, robotics as well as control theory. It is dedicated to researchers in science and industry, students, and practicing engineers, who wish to update and enhance their knowledge on modern methods and innovative applications. The authors and editor of this book wish to motivate people, especially under-graduate students, to get involved with the interesting field of robotics and mechatronics. We hope that the ideas and concepts presented in this book are useful for your own work and could contribute to problem solving in similar applications as well. It is clear, however, that the wide area of automation and robotics can only be highlighted at several spots but not completely covered by a single book.

\title{
How to reference
}

In order to correctly reference this scholarly work, feel free to copy and paste the following:

Laura Celentano (2008). An Innovative Method for Robots Modeling and Simulation, New Approaches in Automation and Robotics, Harald Aschemann (Ed.), ISBN: 978-3-902613-26-4, InTech, Available from: http://www.intechopen.com/books/new_approaches_in_automation_and_robotics/an_innovative_method_for_r obots_modeling_and_simulation

\section{INTECH}

open science | open minds

\section{InTech Europe}

University Campus STeP Ri

Slavka Krautzeka 83/A

51000 Rijeka, Croatia

Phone: +385 (51) 770447

Fax: +385 (51) 686166

www.intechopen.com

\section{InTech China}

Unit 405, Office Block, Hotel Equatorial Shanghai

No.65, Yan An Road (West), Shanghai, 200040, China

中国上海市延安西路65号上海国际贵都大饭店办公楼405单元

Phone: +86-21-62489820

Fax: +86-21-62489821 
(C) 2008 The Author(s). Licensee IntechOpen. This chapter is distributed under the terms of the Creative Commons Attribution-NonCommercialShareAlike-3.0 License, which permits use, distribution and reproduction for non-commercial purposes, provided the original is properly cited and derivative works building on this content are distributed under the same license. 